\title{
Performance Evaluation of Audio Codecs using VolP Traffic in Wireless LAN using RSVP
}

\author{
Priyanka Luthra \\ M.Tech CSE \\ D.A.V.I.E.T, Jalandhar
}

\author{
Manju Sharma \\ Associate Professor IT \\ D.A.V.I.E.T, Jalandhar
}

\begin{abstract}
VoIP has become an interesting topic of research in both the internet and the telecommunication industry. The tremendous increase in popularity of VoIP services is a result of huge growth in broadband access. In wired as well as in wireless communication, VoIP is expected to completely replace the traditional telephony approaches. To provide a good quality speech through VoIP applications, certain QoS parameters must be analyzed. These QoS parameters help us to evaluate the performance of various networking protocols, voice encoding schemes, etc. Numbers of QoS techniques like IntServ, DiffServ and RSVP are adopted to ensure good quality in IP based networks. In this paper, our main contribution is to analyze and evaluate the performance of various voice encoding schemes using RSVP, in a VoIP based wireless LAN. The network model designed in this paper is based on OPNET IT GURU Academic Edition. Various scenarios for different voice encoding schemes using RSVP are setup in the OPNET simulation environment. Different parameters that indicate the QoS like throughput, end to end delay, delay variations, traffic send, traffic received, etc. are calculated and analyzed in WLAN.
\end{abstract}

\section{Keywords}

VoIP, WLANs, QoS, IntServ, DiffServ, RSVP.

\section{INTRODUCTION}

In the recent years, there has been significant increase in the area of wireless networking. Wireless Local Area Networks (WLANs) have made a huge impact on the telecommunication environment. Corporations are turning towards WLANs to network their offices and campuses. Even, most of consumers are increasingly installing WLAN equipment in their homes to network their home computers, laptops and appliances. Furthermore, there is a rush to populate public spaces and commercial areas with WLAN coverage. WLANs are relatively easy to install, stable, have acceptable speed, and are inexpensive. WLANs allow some network devices to move from one place to another to extend the network beyond the limits of the cables. Although WLANs are always considered as a good data-centric network choice in colleges, universities, airports, restaurants, and other enterprise markets, there is also a growing interest in using them for multimedia applications. So, the use of wireless networks has extended beyond simple text and data transmission to extremely complex voice and video networks. This led to the existence of VoIP. Voice over Internet Protocol (VoIP) is a term used for delivery of speech or voice over IP networks such as the Internet or other packet-switched networks. VOIP converts the voice signal from your telephone into a digital signal that travels over the internet then converts it back at the other end so you can speak to anyone with a regular phone number. VoIP is simply the transmission of voice conversations over IP-based networks.
Although Internet Protocol was originally designed for data networking, its high usage and effectiveness has led its adaptation to voice networking when placing a VoIP call using a phone with an adapter; you'll hear a dial tone as you listen before with simple telephone set. VoIP may also allow you to make a call directly from a computer using a conventional telephone [1]. A major advantage of VOIP and Internet telephony is that it avoids the tolls charged by ordinary telephone service which results in expensive calls. VoIP telephone calls (voice) bypass the typical publicswitched telephone network and transmit voice calls over a private network. In General Voice over Internet Protocol (VoIP) refers to the use of the Internet for making telephone calls. The VoIP service require its users to pay only their usual Internet connection charges regardless of where they are calling anywhere and anytime in the world. With the transfer of voice data the quality of the voice must keep in mind as the IP is designed to transfer data packets rather than voice packets so QoS has become a critical issue. Also realtime applications like voice, unlike data (non-real time) applications, are very sensitive to delay. Therefore, QoS of VoIP is an important concern to ensure that voice packets are not delayed, lost or dropped during the transmission over the network. A VoIP service has to provide its users with good quality of services, the same as the quality of PSTN services or even better. Therefore, VoIP applications require a strong QoS for the satisfaction of their users. VoIP quality of service is measured based on different parameters like delay, jitter, packet loss, throughput and echo. VoIP QoS is improved by controlling the values of these parameters to be within the acceptable range. In the next section various QoS factors related to voice transmission are defined.

\section{QOS PARAMETERS}

There are many parameters which help us to verify the Quality of a VoIP application. Some of these QoS Parameters are:

Bandwidth: The bandwidth for VoIP application is the key for voice quality. Bandwidth is interchangeably used with connection speed, although technically they are not exactly the same. Bandwidth is in fact a range of frequencies through which data is transmitted. Bandwidth dependency is besides one of the main drawbacks of VoIP.

Throughput: Throughput refers to how much data can be transferred from source to destination in a given amount of time. It depends upon the bandwidth also. Throughput is a measure of data rate (bits per second) generated by the application.

Retransmission Attempts: There may be failure of packets occur in the transmission process due to different factors like noise etc., so retransmission of packets needed for accurate 
delivery, but this retransmission is fixed and if no successful delivery of packets occur for that no of time the packet will result out to be dropped.

Fixed and Variable delay: There are two distinct types of delay called fixed and variable. Fixed delay components add directly to the overall delay on the connection. Fixed-network delay is introduced by network when the electrical and optical signals travel the media en route to the receiver. For high networks which include many transit nodes for inter-region interconnections, network delay can be very large, so is necessary to use coding scheme with lower coding/decoding delay. Variable delays arise from queuing delays in the buffers on the serial port connected to the WAN. These buffers create variable delays, called jitter, across the network. The congestion affects the overall latency of a packet in transit from source to destination. Applying QoS mechanisms does affect the variable-network delay.

End to End Delay: In general end-to-end delay is the length of time taken for the quantity of interest to reach its destination. In computer networks, end-to-end delay is the amount of time it takes for the head of the signal to travel from the sender to the receiver over a medium. When end-toend delay reaches about 150 milliseconds, participants in a telephone conversation begin to notice its effects. End-to-end delay above 400 milliseconds can make normal conversations impossible. Delay affects the quality of a conversation without affecting the actual sound of the voice signal - delay does not introduce noise or distortion into the voice channel.

Network Load: Employing the number of retransmission of packets may increase the load of the network. So the network load defines the total load on WLAN including the load of the application and the retransmission. Different applications have different effect on network load. Network load also depends upon the type and size of data. The real time applications like voice and video data the load will be high as compare to other type of data.

Clarity: Clarity generically refers to a voice signal's fidelity, clearness, lack of distortion, and intelligibility. In a VoIP environment, clarity problems are caused by delay, packet loss and uncontrolled jitter. Clarity is also significantly impacted by the codecs used on the voice channel.

Codec is a term used for conversion of analog signals to digital form for transmission and from digital to analog form. This is the term used for the word coder-decoder. There are many codecs for audio, video, fax and text. The most common codecs used for VoIP applications are G.711, G.722, G.723, G.726, G.729, etc. We used in our evaluations some of G.7xx of ITU-T standards for audio compression and decompression.

\section{VOIP CODECS}

Codec is a term used for conversion of analog signals to digital form for transmission and from digital to analog form. This is the term used for the word coder - decoder. There are many codecs available for audio, video and text. The most common codecs used for VoIP applications are G.711, G.722, G.723, G.726, G.729, etc. We used in our evaluations some of G.7xx of ITU-T standards for audio compression and decompression.
Table 1. Common Audio Codecs

\begin{tabular}{|c|c|l|}
\hline Codec & $\begin{array}{c}\text { Bandwidth } \\
\text { /kbps }\end{array}$ & Comments \\
\hline G.711 & 64 & $\begin{array}{l}\text { Delivers precise speech } \\
\text { transmission. Very low } \\
\text { processor requirements. }\end{array}$ \\
\hline G.722 & $48 / 56 / 64$ & $\begin{array}{l}\text { Adapts and } \\
\text { compressions varying } \\
\text { bandwidth conserved for } \\
\text { network congestion. }\end{array}$ \\
\hline G.723.1 & $5.3 / 6.3$ & $\begin{array}{l}\text { High compression with } \\
\text { high quality audio. Can } \\
\text { use with dial-up. Lot of } \\
\text { processor power. }\end{array}$ \\
\hline G.726 & $16 / 24 / 32 / 40$ & $\begin{array}{l}\text { An improved version of } \\
\text { G.721 and G.723 } \\
\text { (different from G.723.1) }\end{array}$ \\
\hline G.729 & 8 & $\begin{array}{l}\text { Excellent bandwidth } \\
\text { utilization. Error tolerant. }\end{array}$ \\
\hline & $\begin{array}{l}\text { High compression ratio. } \\
\text { Free and available in } \\
\text { many hardware and } \\
\text { software platforms. Same } \\
\text { encoding is used in GSM } \\
\text { cellphones }\end{array}$ \\
\hline
\end{tabular}

The table shows number of compression schemes but the most commonly used audio codec is the G.711 for VoIP applications.

G.711 PCM codec is the most famous codec among all the codecs. G.711 represents logarithmic pulse-code modulation (PCM) with 8 bits samples for signals of voice frequencies, sampled at the rate of 8000 samples/second, on $64 \mathrm{kbps}$ channel. Using G.711 audio codec for VoIP will give the best voice quality; as it uses no compression and it is the same codec used by all Public Switched network and ISDN lines. It sounds just like using a regular phone or ISDN phone. But this codec takes more bandwidth then other codecs, up to 84 Kbps including all TCP/IP overhead. However, with increasing broadband bandwidth, this should not be a problem.

The next audio codec is G.722. It is an ITU standard codec that provides $7 \mathrm{kHz}$ wideband audio at data rates in different range from 48, 56 and $64 \mathrm{Kbit} / \mathrm{s}$. It provides better speech quality over older narrowband codecs such as G.711, without much complexity.

G.723.1 is the ITU-T standard that specifies the coded representation for speech in PSTN using Algebraic CodeExcited Linear Prediction (CELP) coding rates at $5.3 \mathrm{Kbit} / \mathrm{s}$ and Multipulse Maximum Likelihood Quantization (MPMLQ) at $6.3 \mathrm{Kbit} / \mathrm{s}$. The $6.3 \mathrm{Kbit} / \mathrm{s}$ provides very good voice quality whereas the lower bit rate provides good quality with some more functionality.

G.726 is the Recommendation for speech coding at 40, 32, 24, and $16 \mathrm{Kbit} / \mathrm{s}$ (variable bit rates) using Adaptive Differential Pulse Code Modulation (ADPCM) transcoding technique.

G.278 is the ITU-T Recommendation for speech coding at 16 Kbit/s utilizing Low-Delay Code-Excited Linear Prediction Coding (LD-CELP).This codec provides better voice quality using less bandwidth but the quality become poorer in the presence of background traffic.

G.729 is an ITU standard offers toll quality speech at a low bit rate of $8 \mathrm{Kbps}$ using CS-ACELP (Conjugate Structure Algebraic Code Excited Linear Prediction). However, it is a rather "costly" codec in terms of CPU processing time; 
therefore some VoIP phones and adapters can only handle one G.729 call (channel) at a time. This codec provides robust performance but at the price of its complexity. This can cause calls to fail if the user attempts to use three-way calling, or place simultaneous calls on both lines of a two-line device, and G.729 is the only allowed codec.

Another standard used in our evaluations is ETSI GSM. The GSM system uses Linear Predictive Coding with Regular Pulse Excitation (LPC-RPE codec). It is a full rate speech codec and operates at $13 \mathrm{Kbits} / \mathrm{sec}$. As a comparison, the old public telephone networks use speech coding with bit rate of $64 \mathrm{Kbit} / \mathrm{s}$.

\section{THE E-MODEL}

The E-model is an analytic model defined in the ITU-T recommendation G.107 and some other associated ITU-T recommendations; it provides a framework for an objective on-line quality estimation based on network performance measurements (e.g., delay and loss) and application level factors. In simple words it is a tool that helps us to predict the voice quality during the telephonic conversation. The E-model assesses the combined effects of varying transmission parameters that affect the conversation quality of narrow band telephony. The E-model consider impairments like telephonyband impairments, impairments due to low bit coding devices and telephony impairments like loss, noise and delay. The principle of the E-model is based on the assumptions that these transmission impairments can be transformed into psychological factors [3]. The primary output of the E-model is a transmission rating factor $R$ :

$\mathrm{R}=\mathrm{Ro}-\mathrm{Is}-\mathrm{Id}-\mathrm{Ie}-\mathrm{A} \quad$ where:

Ro = basic Signal-to-noise ratio (SNR), in absence of any impairments

Is = impairments occurred simultaneously (connection loudness, quantization distortion)

Id = impairment due to delay of voice signal

$\mathrm{Ie}=$ impairment due to compression techniques (loss due to very low bit-rate encoding) and loss

A = user tolerance of some degradation due to special situations (mobility, hard-to-reach locations)

The value of $R$ lies between 0 to 100 . The value 100 means excellent and the value 0 means poor. The $\mathrm{R}$-value calculation start with the basic signal that is if there is no network, no equipment then the quality is perfect but the network and equipment effect the signal and lowers down the quality of the signal.

The R-factor can be further translated into MOS (Mean Opinion Score). ITU-T has defined the methodology of determining how satisfactorily given telephone connections and their communications may be expected to perform. The methods described by this recommendation are intended to be generally applicable for any possible form of degradation: loss, circuit noise, transmission errors, environmental noise, talker echo, distortion due to encoding, etc. The evaluation procedure is based on subjective tests. The following values are assigned depending on the quality of the connection:

Excellent $=5$ Good $=4$ Fair $=3$ Poor $=2 \mathrm{Bad}=1$

R-factor can be modeled into MOS scale through this expression:

$M O S=1+0: 035 R+7: 10 ; 6 R(R ; 60)(100 ; R)$

The relationship between R-value, MOS and user quality are shown in the table.
Table 2. R-value/MOS Relationship.

\begin{tabular}{|l|l|l|}
\hline $\begin{array}{l}\text { R-value } \\
\text { range }\end{array}$ & MOS range & Quality \\
\hline $90-100$ & $4.34-5.00$ & Best \\
\hline $80-90$ & $4.03-4.34$ & High Satisfied \\
\hline $70-80$ & $3.60-4.03$ & Medium \\
\hline $60-70$ & $3.10-3.60$ & Low \\
\hline$<60$ & $<3.1$ & Poor \\
\hline
\end{tabular}

\section{QoS Techniques}

There are two main techniques that have been developed to provide Quality of Service to the network based applications. These techniques are Differentiated Services (DiffServ) and the Integrated Services (IntServ).

The DiffServ model follows a very flexible approach regarding classifying its datagrams. DiffServ works on provisioned QoS, where network elements are setup to deal with class of different services with different QoS requirements. The data Packets are designated respectively by marking with specific DiffServ Code. DiffServ provides a high level of scalability as it is designed to provide quality services to small well defined set of building blocks and which can further be aggregated. Therefore it is quality management technique which is well suited to manage resources in core networks.

In IntServ, Network resources are explicitly identified and reserved, and datagrams are treated in a per flow manner. Data flow is assigned by imposing explicit reservations on the end-to-end path. IntServ is applied usually in access routers or gateways and serve different services in different ways. The QoS IntServ model defines three classes of services based on the type of application delay requirement. These are guaranteed service class, Controlled-load service class and best effort service class. So the main advantage of IntServ is that it provides various service classes which matches to the different applications and their requirements. The IntServ architecture also specifies extensions to the best-effort traffic model. It defines how applications place reservations. Resource Reservation Protocol (RSVP) is a robust signaling protocol developed to operate within the IntServ model. RSVP is an IETF-defined signaling protocol that uses Integrated Services (Intserv) to convey QoS requests to the network. RSVP is a quality technique used for delay sensitive application like VoIP. This system follows the signaled QoS where end user signal their own QoS needs to the network. In this protocol techniques are defined to relinquish the reserved resources once an RSVP session is terminated. RSVP operation generally results in resources being reserved in each node along the end-to-end path, although it can function through non-RSVP routers along the way [16]. RSVP is a soft state protocol and it maintain state on a per-flow basis but on the same time it requires periodic refreshment by sending refresh message. If it does not receives the require refresh message it tears down the reservation. The end user or host can also send the tear down message to end up the conversation. Customer networks generally include heterogeneous media, including Ethernet or token ring local area network (LAN) media, WLANs, WANs made up of low and high-speed leased lines, modem links, and ATM technology. RSVP also bridges the gap between various applications, the operating system, and media-specific QoS mechanisms. This enables RSVP to send QoS messages structured in media-independent terms, making it highly 
effective signaling protocol for end-to-end QoS over networks that combine different types of low-layer media. Our network model uses the RSVP technique to lower down the network delays as the VoIP applications are highly delay sensitive applications. By using RSVP we can reduce the amount of various delays occurring in our application.

\section{NETWORK MODEL DESCRIPTION}

Our simulation approach uses OPNET IT Guru 9.1 for network modeling. OPNET IT Guru is a powerful communication system simulator developed by OPNET Technologies. OPNET IT Guru 9.1 assists with the testing and design of communications protocols and networks by simulating network performance for wired and/or wireless environments. The OPNET tool provides a hierarchical graphical user interface for the definition of network models. It provides a Virtual Network Environment that models the behavior of an entire network, including its routers, switches, protocols, servers, workstations, different kinds of links and individual applications OPNET IT Guru provides us a vast set of models which helps us simulating the real time applications in a very effective way.

The simulation model for the network under study is illustrated in Figure1. The Application Config, Profile Config and QoS Attribute Config are included in the model. The Wireless LANs has been considered as the components of the whole network and its nodes are using VoIP services. The other components of this network are two generic routers, two switches, FTP server, database server web browsing server, a VoIP Gateway and 3 Departments which are represented by Wireless LAN subnets. Each Wireless LAN subnet contains an Access Point and number of wireless nodes. All these wireless nodes use VoIP services. The Access Point is represented by a wlan_ethernet_router_adv and VoIP nodes are represented by wlan_wkstn_adv. All the network elements have been connected using 100 Base-T links. Figure 1 shows the described topology.

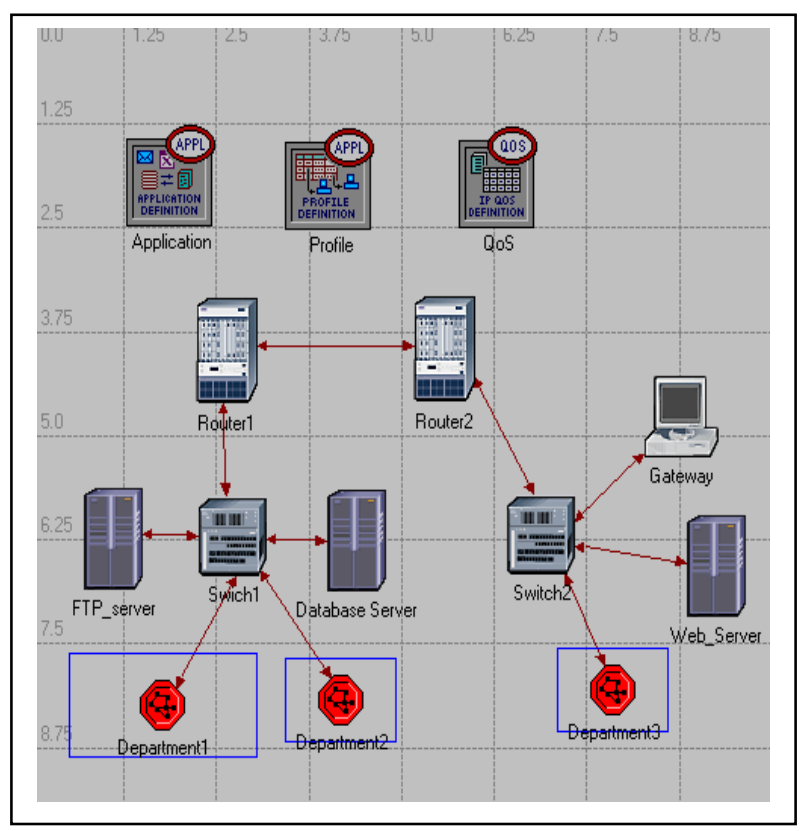

Figure 1: The Network Model

There are 3 scenarios present in our simulation each represents different codec format but the network topology is same as represented in Figure 1.
Figure 2 shows the topology of department 1 Wireless LANs that enclose a Wireless router used as Access point and designated wireless LAN workstations used to model the various activities of the users. Two of these workstations generate works as the voice calling server and rest in the subnet act as parties in VoIP sessions.

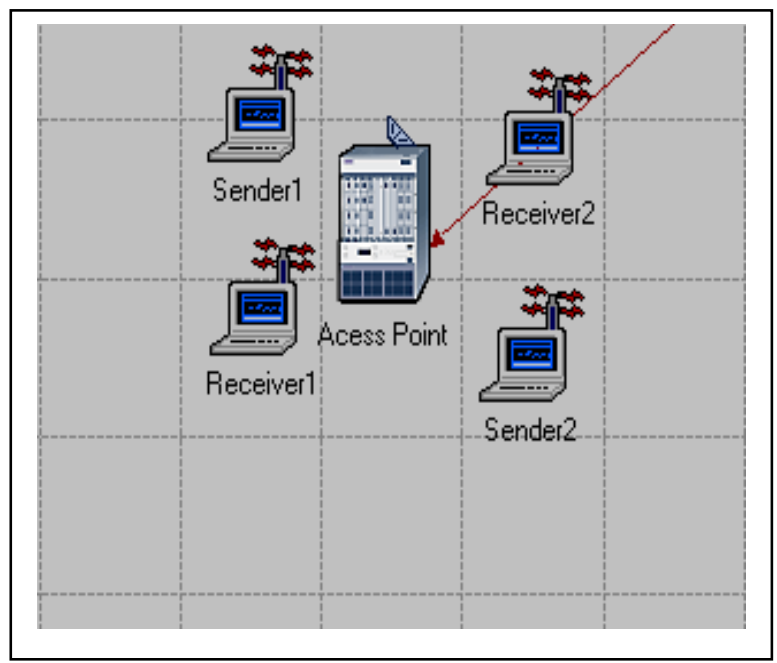

Figure 2: The Wireless LAN subnet for department 1.

In Figure 2 there are 4 wireless workstation nodes and 1 Access Point. Two of the wireless workstation nodes act as voice calling nodes and two of them are the voice called nodes. Sender 1 and Sender 2 are voice calling nodes and Receiver 1 and Receiver 2 are voice called nodes.All these network nodes involved in voice traffic delivery are configured to support resource reservation by means of RSVP.

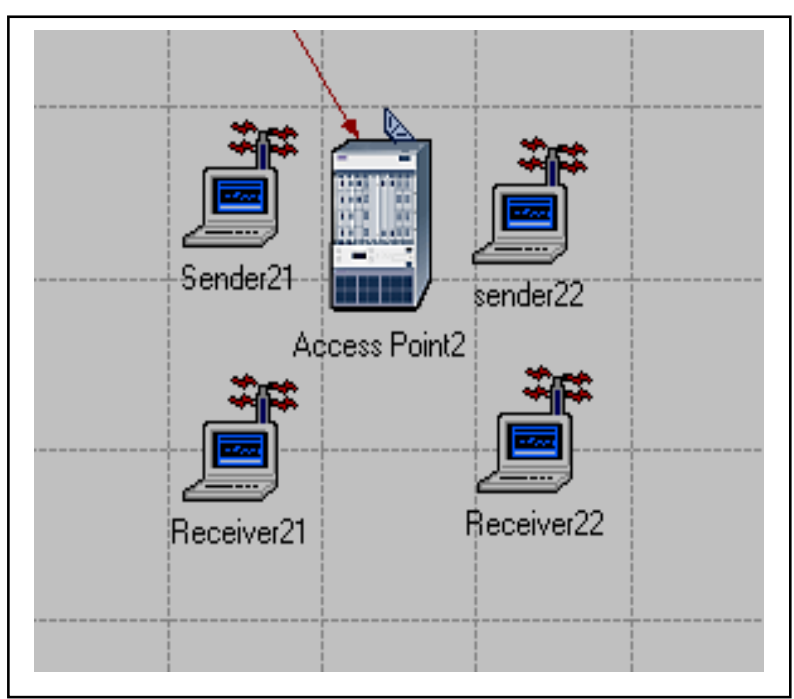

Figure 3: The Wireless LAN subnet for department 2.

In Figure 3 Sender 21 and Sender 22 are voice calling nodes and Receiver 21 and Receiver 22 are voice called nodes. All these VoIP nodes and Access Point are configured to support resource reservation by means of RSVP. 


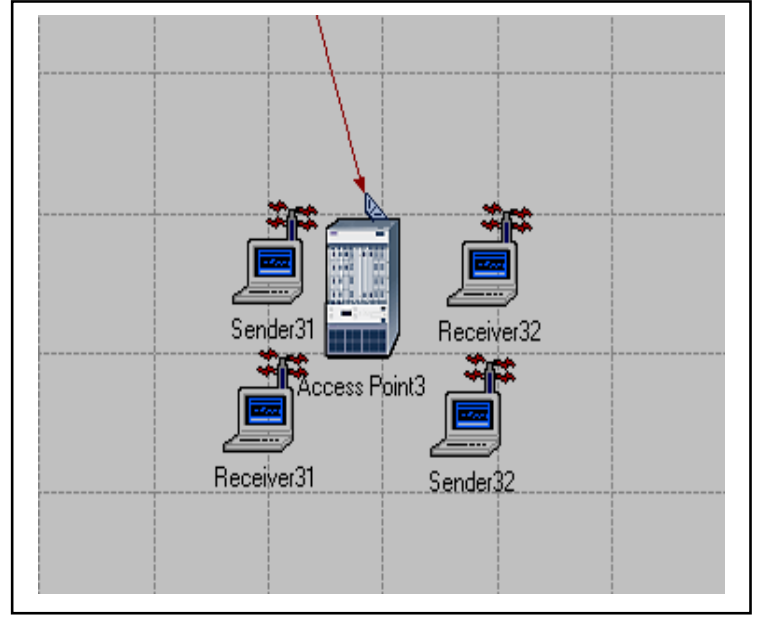

Figure 4: The Wireless LAN subnet for department 3.

In Figure 4 Sender 31 and Sender 32 are voice calling nodes and Receiver 31 and Receiver 32 are voice called nodes. All nodes of this subnet are configured to support resource reservation by means of RSVP.

All the wireless LAN's in our network follows the same structure and all the scenarios follows the same topology. The basic difference in all the scenarios is with the configuration of the corresponding application and the profiles. As one scenario is implemented with the codec scheme G.711, another with the codec scheme G.723.1, and the third with G.729.1 codec scheme. Various comparisons have been performed to find the values of various parameters.

\section{TABLES}

There are 4 applications used in each scenario. These are RsvpVoIP, database, FTP and web browsing applications. The RsvpVoIP application is our Voice over IP application with RSVP enabled. The database, FTP and web browsing applications are configured with low traffic load. Table 1 shows the application description table.

\section{Table 3: Application Description.}

\begin{tabular}{|l|l|}
\hline Applications & Load \\
\hline RsvpVoIP & .... \\
\hline FTP & Low \\
\hline Database & Low \\
\hline Web Browsing & Low \\
\hline
\end{tabular}

In the RsvpVoIP application, voice attribute must be configured as codec scheme G.711, G.723.1, G.729 for Scenario 1, Scenario 2 and Scenario 3 respectively. Along with the voice attributes settings RSVP parameters must be configured. The RSVP parameters are described in Table 4.

\section{Table 4: RSVP Parameters Description.}

\begin{tabular}{|l|l|}
\hline Attribute & Value \\
\hline RSVP Status & Enabled \\
\hline Outbound Flow & Rsvp_flow \\
\hline Inbound Flow & Rsvp_flow \\
\hline
\end{tabular}

RSVP flow is defined in the QoS Attribute Config description. The RSVP flow specifications are described in Table 5.

Table 5: RSVP Flow Specification Parameters.

\begin{tabular}{|l|l|}
\hline Attribute & Value \\
\hline Name & Rsvp_flow \\
\hline Bandwidth(packets/sec) & 50,000 \\
\hline Buffer Size(bytes) & 10,000 \\
\hline
\end{tabular}

All the parameters of wireless LAN must have the same value in the same subnet. The wireless LAN attributes are described in Table 6 .

Table 6: Wireless LAN Parameters.

\begin{tabular}{|l|l|}
\hline Attribute & Value \\
\hline Data rate(bps) & $11 \mathrm{Mbps}$ \\
\hline Physical characteristics & Direct Sequence \\
\hline Buffer size(bits) & 1024000 \\
\hline
\end{tabular}

\section{RESULTS}

The following graphs are obtained after collecting statistics on OPNET IT GURU. The graph gives a comparative picture of the three scenarios. All the three scenarios are using RSVP and each RSVP applications are configured with WFQ weighted fair queuing for resource allocation and execution of packets. The result shows the impact of different voice encoder schemes on different parameters using reservation protocol RSVP in a Wireless Lan based network. As VoIP is delay sensitive application the main aim is to analyze the effect of RSVP on the various delays in a VoIP application. Following are the graphs that show different QoS parameters like throughput of voice application using different voice schemes, end to end delay of voice packet transmissions, network load using different codecs.

\section{A. Traffic Received (packets/sec)}

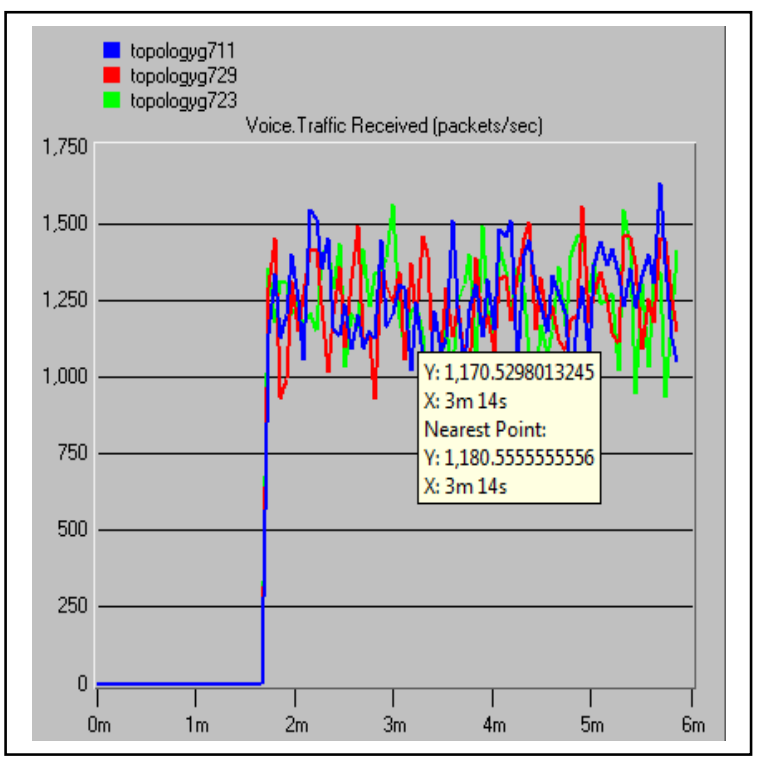

Figure 5: Traffic Received (packets/sec) 
In Figure 5 Traffic received for g.711 is more than g.729 and g.723.1 as g.711 is heavy bandwidth audio codec. After simulating for 6 minutes the average packets received in case of g.711 is 886 whereas in case of g.729 is 875 and in case of g.723.1 is 878 . The traffic received for g.723.1 codec lies between $\mathrm{g} .711$ and g.729.

\section{B. End to End Delay (sec)}

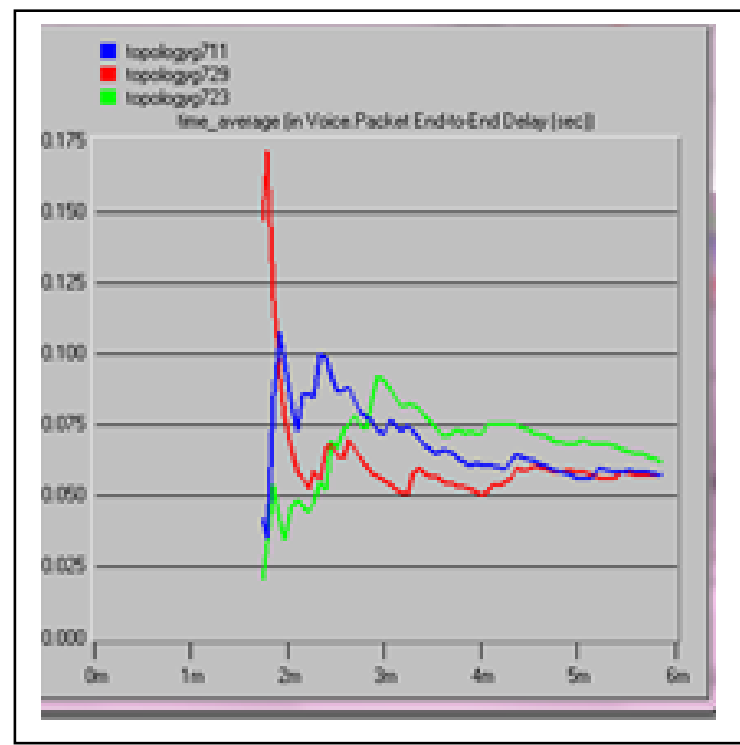

Figure 6: End to End Delay (sec)

Figure 6 describe the End to End delay of the codec g.729 starts with the maximum but drop down to minimum throughout the simulation run. The end to end delay in case of codec g.723.1 is lesser in the starting of simulation but gradually it reaches slightly higher than the other two codecs. The end to end delay in case of g.711 lies in between the other two audio codecs.

\section{Wireless LAN Delay (sec)}

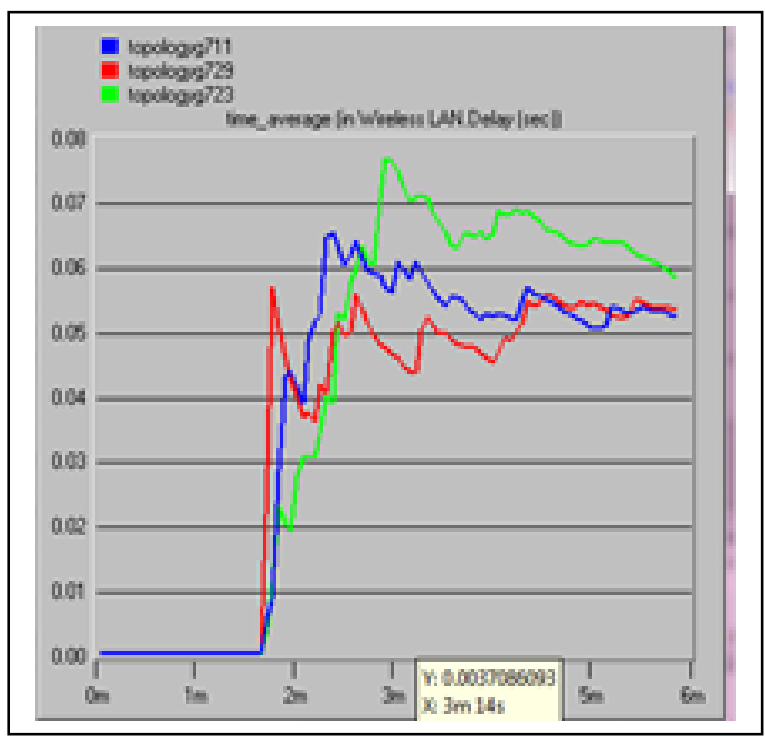

Figure 7: Wireless LAN Delay (sec)
Figure 7 describe the Wireless LAN delay of the codec g.729 is minimum. The Wireless LAN delay in case of codec g.723.1 is higher than the other two codecs. The delay of g.723.1 is the highest at the early stage but after some time it fall down. The Wireless LAN delay in case of g.711 lies in between the other two audio codecs.

\section{Media Access Delay (sec)}

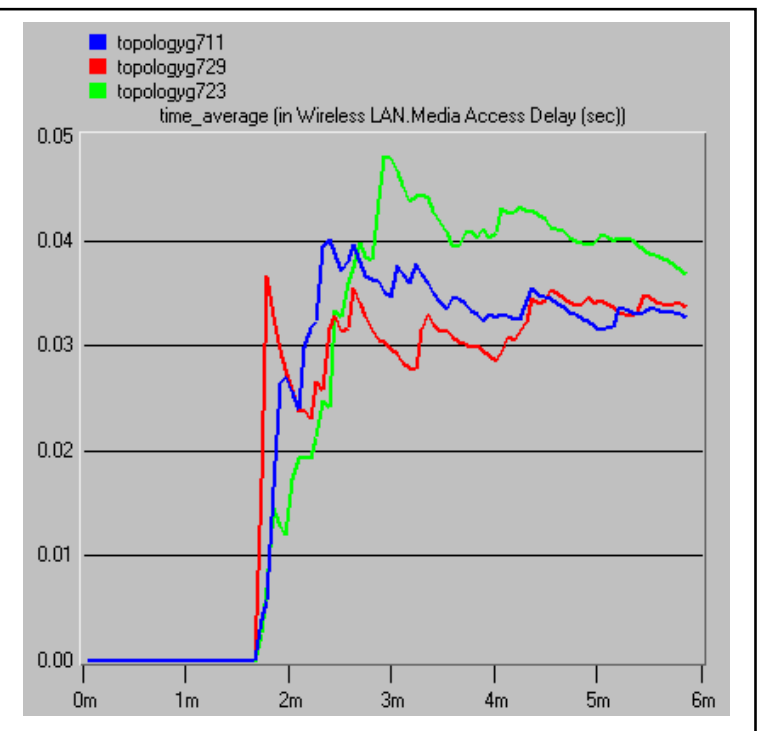

Figure 8: Media Access Delay (sec)

Figure 8 describe the Media Access delay of the codec g.729 is minimum. The Wireless LAN delay in case of codec g.723.1 is much higher than the other two codecs. The Wireless LAN delay in case of g.711 lies in between the other two audio codecs. Media access delay of g.723.1 is the maximum in the initial level of simulation and falls down in the later stage.

\section{E. Wireless LAN Delay Variation (sec)}

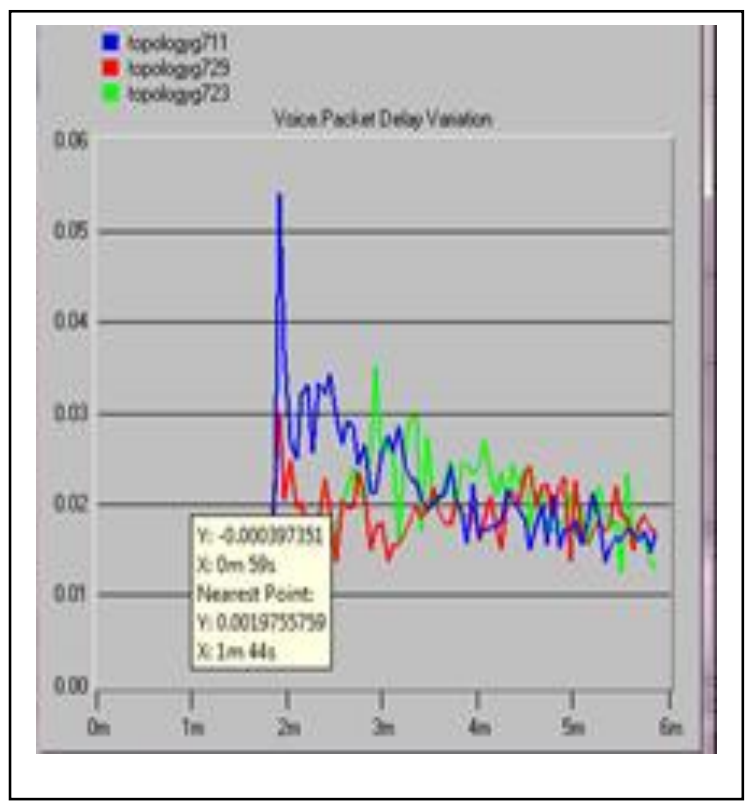

Figure 9: Wireless LAN Delay Variation (sec) 
Figure 9 describe the wireless LAN delay variation of the codec g.729 is minimum and approximately constant throughout the simulation. The wireless LAN delay variation in case of codec g.711 is higher than the other two codecs in the starting of the simulation but the variations falls gradually as the simulation goes forward. The Wireless LAN delay in case of g.723.1 lies in between the other two audio codecs. As constant delay variations leads to smooth communication as in case of g.729 so codec g.729 gives better results than audio codecs g.711 and g.723.1.

\section{CONCLUSION}

The results in this paper compare three audio codecs g.711, g.723.1 and g.729 using RSVP services. VoIP is a delay sensitive application so the main aim is to reduce the delay of these codecs in VoIP application by implementing RSVP and checking the performance of the audio codecs by evaluating QoS parameters. Also the queuing technique used for RSVP application is WFQ as weighted fair queuing and RSVP both together results in lesser delays and better throughput. The result shows that by using RSVP the delay like end to end delay, media access delay or wireless LAN delay is lesser in codec g.729 as compared to the other two referenced codecs g.711 and g.723.1. Also the delay variation in case of codec g.729 is almost constant and quite less as compared to g.711 and g.723.1. The lesser the delay variation better will be the quality of the voice.

\section{REFERENCES}

[1] Voice Over Internet Protocol (VoIP) at: http://www.fcc.gov/guides/voice-over-internet-protocolvoip.

[2] Navdeep Singh Chauhan, Loveljeet Kaur, "Evaluation of QoS for Different Voice Schemes in Wireless LAN using OPNET Modular", Int. J. on Recent Trends in Engineering \& Technology, Vol. 05, No. 01, Mar 2011.

[3] Eduard-Cristian Popovici, Tatiana Radulescu "Coding scheme impact on the IP-QoS network utilization and voice quality" 8th COST290 Management Committee and Technical Meeting, Malaga, Spain, February 15-16, 2007.

[4] E Aboelela, Morgan Kaufmann, "Network simulation Experiments Manual”, 2003, ISBN:0-12-042171-2

[5] Ajay Shreshtha, Khaled: "Investigating the Effects of Encoder Schemes, WFQ \& SAD on VoIP QoS", University of Bridgeport 2010.

[6] IT Guru Academic Edition. OPNET Technologies, (2007) http:// www.opnet.com/university program/ itguru academic_edition.

[7] J. Theunis \& B. Van den Broeck. "OPNET in Advanced Networking Education”. Retrieved April 10, 2003.
[8] Kumar Manoj, Parmanand, S. C. Sharma \& S.P. Singh “ Performance of QOS parameters in wireless Ad-Hoc networks", Proceedings of the World Congress on Engineering and Computer Science 2009, vol 1 WCECS 2009, October 20-22, 2009, San Francisco, USA.

[9] Srinivas Vegesna: "IP Quality of Service", Cisco Press, 2003.

[10] Tim Szigeti, Christina Hattingh: "End-to-End QoS Network Design: QoS in LANs, WANs and VPNs", Cisco Press, 2005.

[11] J. Theunis, B. Van den Broeck, P. Leys, J. Potemans1, E. Van Lil, A. Van de Capelle, "OPNET in Advanced Networking Education “, Katholieke Universiteit Leuven, Division ESAT-Telemic.

[12] A. Al-Naamany, H. Bourdoucen and W. Al-Menthari, "Modeling and Simulation of Quality of Service in VoIP Wireless LAN", Journal of Computing and Information Technology - CIT 16, 2008, 2, 131-142

[13] Vineet Grewal, Ajay k sharma, "On Performance Evaluation of Different QoS Mechanisms and AMC scheme for an IEEE 802.16 based WiMAX" International Journal of Computer Applications (0975 8887) Volume 6- No.7, September 2010.

[14] Haniyeh Kazemitabar, Sameha Ahmed, Kashif Nisar, Abas B Said, Halabi B Hasbullah," A comprehensive review on VoIP over Wireless LAN networks", Department of Computer \& Information Sciences Universiti Teknologi PETRONAS Bandar Seri Iskandar, 31750 Tronoh, Perak, Malaysia.Vol. 2 (2) - September 2010

[15] Rizik Al-Sayyed, Colin Pattinson, and Tony Dacre," VoIP and Database Traffic Co-existence over IEEE 802.11b WLAN with Redundancy", World Academy of Science, Engineering and Technology 262007.

[16] Ajith Kumar V. 1 and Sheela Ganesh Thorenoor," Analysis of IP Network for different Quality of Service", 2009 International Symposium on Computing, Communication, and Control (ISCCC 2009) Proc .of CSIT vol.1 (2011) @ (2011) IACSIT Press, Singapore.

[17] Mohammad Mirza Golam Rashed and Mamun Kabir, "A comparative study of different queuing techniques in VoIP, video conferencing and file transfer". Daffodil international university journal of science and technology, volume 5, issue 1, January 2010.

[18] Rossitza Goleva, Maria Goleva, "VOIP traffic shaping analysis in Metropolitan Area Networks". International Journal Information Technologies and Knowledge, Vol 2, 2008 . 